\title{
Diagnostic et traitement du syndrome de détresse respiratoire aiguë
}

\author{
Shannon M. Fernando MD MSc, Bruno L. Ferreyro MD, Martin Urner MD, Laveena Munshi MD MSc, \\ Eddy Fan MD PhD
}

Citation : CMAJ 2021 May 25;193:E761-8. doi : 10.1503/cmaj.202661-f

Voir la version anglaise de l'article ici : www.cmaj.ca/lookup/doi/10.1503/cmaj.202661

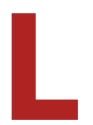

e syndrome de détresse respiratoire aiguë (SDRA) est une forme gravissime d'insuffisance respiratoire, caractérisée par une atteinte pulmonaire inflammatoire diffuse aiguë ${ }^{1}$ qui entraîne une augmentation de la perméabilité des capillaires alvéolaires et un œdème pulmonaire non hydrostatique. Sur le plan clinique, le SDRA se manifeste par une hypoxémie et une détresse respiratoire marquées; les patients progressent souvent vers une insuffisance respiratoire qui nécessite une ventilation mécanique effractive à l'unité de soins intensifs (USI). Le risque de mortalité est élevé. Divers problèmes de santé peuvent causer le SDRA, notamment la pneumonie, le sepsis extrapulmonaire ou le choc septique, les traumas et la pancréatite. Malgré l'existence de lignes directrices consensuelles sur la prise en charge du SDRA ${ }^{2-4}$, celle-ci continue de varier substantiellement dans le monde, et on constate un manque de données probantes sur le sujet, y compris dans le contexte de la COVID-195,6. Nous fournissons ici une mise à jour sur le diagnostic et le traitement du SDRA pour les cliniciens généralistes, en nous fondant sur de récentes lignes directrices de pratique clinique, des revues systématiques et des études originales (encadré 1 ).

\section{Encadré 1 : Données utilisées pour la présente revue}

Nous avons procédé à une interrogation ciblée de MEDLINE, Embase et la base des revues systématiques Cochrane, de leur création jusqu'au 20 février 2021, pour y recenser les essais randomisés et contrôlés, les revues exploratoires, les revues systématiques, les méta-analyses et les guides de pratique clinique. Nous avons utilisé les mots clés « acute respiratory distress syndrome " [syndrome de détresse respiratoire aiguë], "ARDS » [SDRA], « respiratory failure » [insuffisance respiratoire] et « acute lung injury » [atteinte pulmonaire aiguë]. Nous avons poursuivi notre recherche en examinant les références des articles retenus et complété notre démarche par une interrogation de nos propres collections.

\section{POINTS CLÉS}

- Le syndrome de détresse respiratoire aiguë (SDRA) peut avoir diverses étiologies et se manifeste sur le plan clinique par une insuffisance respiratoire hypoxémique aiguë, caractérisée par une atteinte pulmonaire inflammatoire diffuse aiguë entraînant une augmentation de la perméabilité des capillaires alvéolaires et un œdème pulmonaire non hydrostatique.

- Le diagnostic clinique du SDRA repose sur la définition de Berlin; la gravité de la maladie et le pronostic de survie sont fonction du degré d'hypoxémie.

- Le traitement du SDRA repose principalement sur la ventilation mécanique effractive associée à une stratégie de protection pulmonaire, mais d'autres traitements d'appoint ont fait l'objet d'essais, avec divers degrés d'efficacité.

- Sur les plans clinique et radiologique, la COVID-19 grave ressemble au SDRA; sa prise en charge devrait donc suivre les mêmes principes, et tenir compte des conclusions de récents essais randomisés de grande qualité.

\section{Qu'est-ce que le SDRA et comment est-il diagnostiqué?}

Le syndrome de détresse respiratoire aiguë a d'abord été décrit en 1967 comme un syndrome clinique caractérisé par le déclenchement subit d'une tachypnée, d'une hypoxémie et d'une perte de compliance pulmonaire à la suite de divers stimuli; la description originale mentionnait également que le SDRA ne répondait pas aux simples traitements respiratoires habituels ${ }^{7}$. La caractéristique principale de ce syndrome est une inflammation pulmonaire diffuse qui entraîne un œdème. Sur le plan morphologique, la phase aiguë du SDRA est caractérisée par une atteinte pulmonaire diffuse ${ }^{1}$. Des critères diagnostiques formels du SDRA n'ont largement été acceptés qu'après la Conférence de consensus américano-européenne (AECC) de $1994^{8}$. Les critères de l'AECC incluent l'apparition subite d'une hypoxémie, la présence d'infiltrats bilatéraux 


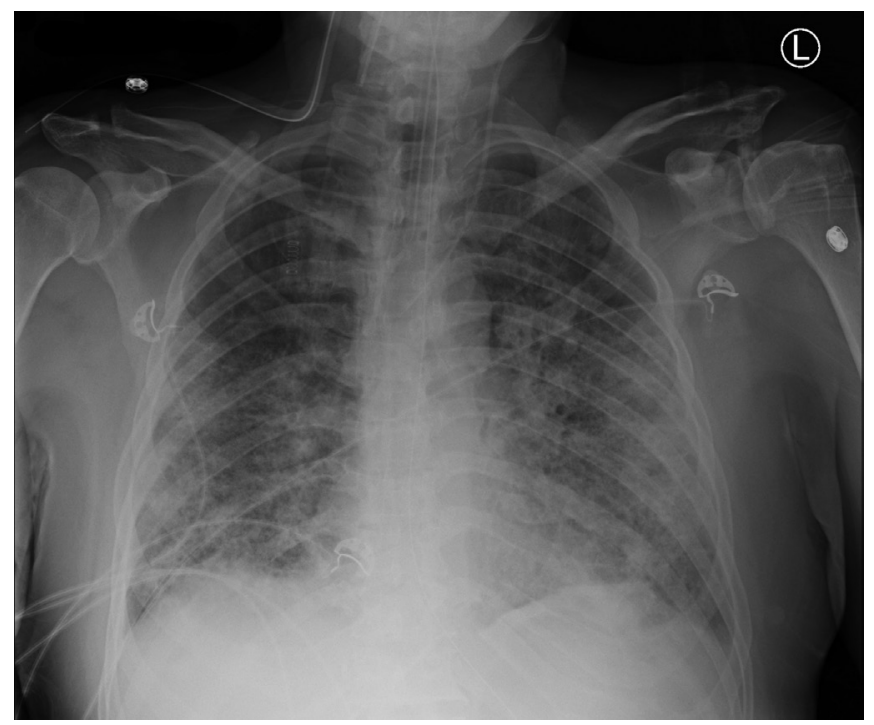

Figure 1 : Radiographie pulmonaire antéropostérieure montrant des infiltrats pulmonaires bilatéraux, qui concordent avec un syndrome de détresse respiratoire aiguë.

non cardiogéniques à la radiographie pulmonaire et l'absence d'hypertension auriculaire gauche. L'hypoxémie était quantifiée en calculant le rapport entre la pression partielle d'oxygène et la fraction d'oxygène inspirée $\left(\mathrm{PaO}_{2} / \mathrm{FiO}_{2}\right)$, un rapport $\mathrm{PaO}_{2} / \mathrm{FiO}_{2}<200 \mathrm{~mm} \mathrm{Hg}$ étant nécessaire pour un diagnostic de SDRA. La définition de l'AECC était limitée par plusieurs facteurs, notamment le manque de données précises sur le moment du déclenchement, la variabilité potentielle de l'interprétation des radiographies pulmonaires entre les observateurs et la nécessité de cathétériser l'artère pulmonaire pour écarter une hypertension auriculaire gauche ${ }^{1}$.

En 2012, les critères diagnostiques cliniques du SDRA ont été précisés afin de tenir compte de ces limites, ce qui a donné la définition de Berlin ${ }^{9}$. Pour qu'on puisse poser un diagnostic de SDRA, le patient doit avoir : des symptômes nouveaux ou exacerbés dans la semaine qui suit une atteinte clinique connue; des opacités bilatérales observables à la radiographie pulmonaire antéropostérieure non dues à des épanchements, à des nodules ou à un collapsus lobaire ou pulmonaire; et une hypoxémie, définie par un rapport $\mathrm{PaO}_{2} / \mathrm{FiO}_{2}<300 \mathrm{~mm} \mathrm{Hg}$ et une pression positive de fin d'expiration minimum $\geq 5 \mathrm{~cm} \mathrm{H}_{2} \mathrm{O}$ ne s'expliquant pas entièrement par une insuffisance cardiaque ou une surcharge liquidienne (figure 1).

La définition de Berlin prévoit aussi des catégories de SDRA mutuellement exclusives selon la gravité du syndrome et le degré d'hypoxémie, soit le SDRA léger $\left(\mathrm{PaO}_{2} / \mathrm{FiO}_{2}: 200-300 \mathrm{~mm} \mathrm{Hg}\right)$, modéré $\left(\mathrm{PaO}_{2} / \mathrm{FiO}_{2}: 100-200 \mathrm{~mm} \mathrm{Hg}\right)$ et grave $\left(\mathrm{PaO}_{2} / \mathrm{FiO}_{2}\right.$ : $<100 \mathrm{~mm} \mathrm{Hg}$ ). Ces catégories correspondent aux pronostics, le taux de mortalité étant proportionnel à la gravité selon les ensembles de données utilisés pour établir les critères ${ }^{9,10}$.

\section{Quel est le fardeau associé au SDRA?}

Durant l'étude de cohortes prospectives LUNG SAFE, la définition de Berlin a été utilisée pour repérer les patients atteints d'un
SDRA dans 459 unités de soins intensifs (USI) de 50 pays sur 5 continents 5 . Dans cette étude, $10,4 \%$ de tous les patients admis à l'USI et $23,4 \%$ des patients ayant eu besoin de ventilation mécanique étaient atteints d'un SDRA. Les causes les plus fréquentes étaient la pneumonie, le sepsis extrapulmonaire, l'aspiration et les traumas. La durée médiane de la ventilation mécanique chez les patients atteints d'un SDRA était de 8 jours (écart interquartile 4-16). Le nombre de décès était substantiel : $39,6 \%$ des patients sont décédés à l'hôpital, et le taux de mortalité augmentait avec la gravité du SDRA (34,9\%, 40,3\% et $46,1 \%$ chez les patients atteints de la forme légère, modérée et grave, respectivement). Les données administratives montrent que même si le taux global de mortalité due au SDRA a diminué au cours des 2 dernières décennies, des disparités liées à la race et au sexe persistent, et la morbidité chez les survivants est considérable ${ }^{11}$. Les survivants du SDRA présentent une faiblesse musculaire et une fatigue importantes qui peuvent persister jusqu'à 5 ans après le congé hospitalier, ce qui entraîne une incapacité fonctionnelle et une baisse de la tolérance à l'effort ${ }^{12,13}$. En outre, les survivants décrivent des séquelles psychologiques, cognitives et économiques majeures associées au SDRA ${ }^{13}$.

\section{En quoi consiste le traitement?}

L'étude LUNG SAFE a observé des variations entre les établissements européens quant à l'utilisation des traitements du SDRA fondés sur des données probantes ${ }^{5}$. Peu de traitements se fondent sur de solides données probantes, mais au cours des 2 dernières décennies, d'importants progrès ont été réalisés dans la prise en charge du syndrome, particulièrement en ce qui concerne la ventilation. Ils ont par la suite été intégrés aux lignes directrices de pratique clinique ${ }^{2-4}$. La figure 2 résume une approche suggérée pour la prise en charge du SDRA.

\section{Ventilation mécanique}

Le traitement du SDRA repose principalement sur la ventilation mécanique associée à une stratégie de protection pulmonaire. Les recommandations des récentes lignes directrices de pratique clinique en ce qui concerne la ventilation mécanique sont résumées dans le tableau 1. L'objectif principal est d'éviter l'atteinte pulmonaire induite par le ventilateur, une forme d'atteinte pulmonaire iatrogène qui aggrave l'inflammation et est associée à une hausse des issues défavorables chez les patients sous ventilation mécanique ${ }^{30}$. L'atteinte pulmonaire induite par la ventilation survient lorsqu'un stress mécanique excessif (p. ex., volume courant élevé) se traduit par une réponse inflammatoire (volotraumatisme) qui peut se propager par la circulation et entraîner une défaillance d'organes éloignés (biotraumatisme). Selon des essais randomisés, une ventilation reposant sur des volumes courants moindres par rapport au poids corporel estimé et sur une pression plateau limitée ont substantiellement réduit le taux de mortalité chez les patients atteints d'un SDRA ${ }^{15,16}$. La ventilation associée à une stratégie de protection pulmonaire se fait par contre au prix d'une possible hypercapnie et de l'acidose qui en résulte, qui sont tolérables si elles sont modestes ${ }^{16}$. 


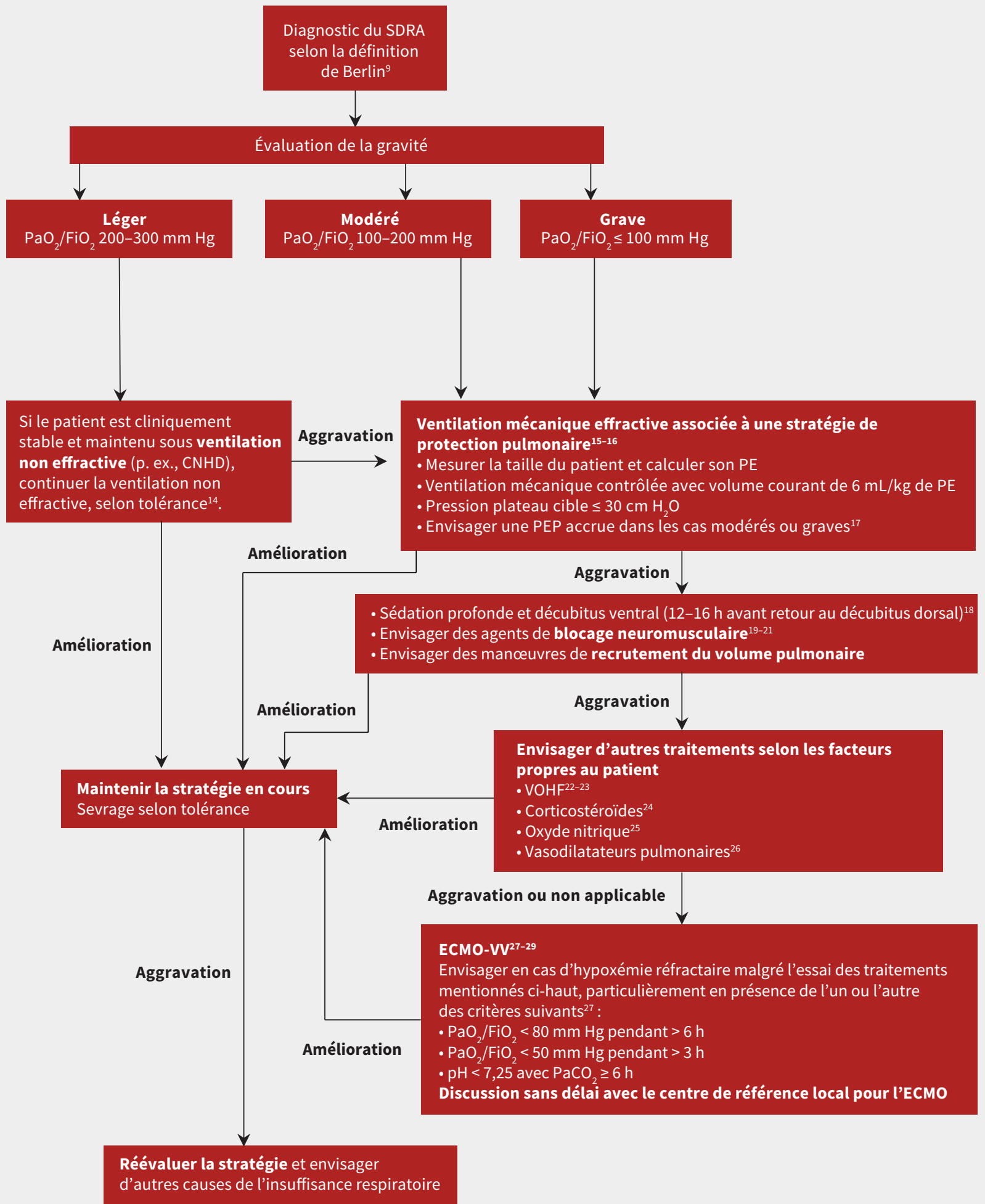

Figure 2 : Algorithme thérapeutique suggéré montrant la stratification du risque et une approche séquentielle pour le traitement des patients atteints du syndrome de détresse respiratoire aiguë (SDRA). Remarque : CNHD = canule nasale de haut débit, ECMO-VV = oxygénation par membrane extracorporelle veino-veineuse, $\mathrm{PE}$ = poids estimé, $\mathrm{PEP}=$ pression expiratoire positive, $\mathrm{VOHF}$ = ventilation oscillatoire à haute fréquence. 
Les lignes directrices existantes suggèrent d'envisager des pressions positives de fin d'expiration plus élevées chez les patients atteints d'un SDRA modéré à grave ${ }^{2-4}$. Le maintien de pressions positives de fin d'expiration plus élevées a l'avantage potentiel de prévenir le collapsus alvéolaire cyclique et les lésions pulmonaires de cisaillement qui en découlent. Toutefois, une pression positive de fin d'expiration trop élevée pourrait également nuire à l'hémodynamie et entraîner une distension pulmonaire excessive. Ce traitement s'est révélé efficace uniquement chez des patients atteints d'un SDRA de modéré à grave ${ }^{17}$.

L'efficacité d'autres méthodes d'amélioration de la ventilation, dont la ventilation oscillatoire à haute fréquence, n'a pas été démontrée $e^{22,23}$, et les lignes directrices déconseillent l'utilisation systématique de cette méthode en particulier chez les patients atteints d'un SDRA ${ }^{2-4}$. La ventilation non effractive pourrait être envisagée chez les patients qui présentent un SDRA léger, mais sera probablement peu utile chez les patients plus gravement atteints ${ }^{14}$. Dans une méta-analyse récente, l'oxygénothérapie par canule nasale à haut débit a permis de réduire le recours à l'intubation et à la ventilation mécanique chez les patients atteints d'insuffisance respiratoire aiguë hypoxémique, mais n'a pas entraîné de baisse du taux de mortalité ${ }^{31}$.

\section{Décubitus ventral}

Il est possible de réduire l'incidence de l'atteinte pulmonaire induite par le ventilateur si l'on place les patients en décubitus ventral. La ventilation mécanique en décubitus dorsal peut entraîner de l'atélectasie et un dérecrutement des régions pulmonaires les plus dépendantes. Le décubitus ventral redistribue les forces mécaniques dans tout le poumon affecté et entraîne une expansion pulmonaire plus homogène et un recrutement des alvéoles des régions pulmonaires dépendantes. Chez des patients atteints d'un SDRA qui ont un rapport $\mathrm{PaO}_{2} / \mathrm{FiO}_{2}<150 \mathrm{~mm} \mathrm{Hg}$, des données probantes de grande qualité montrent que le décubitus ventral réduit le risque de mortalité sans faire augmenter les complications graves ${ }^{18}$. Par conséquent, les lignes directrices préconisent l'utilisation systématique du décubitus ventral chez les patients atteints d'un SDRA grave ${ }^{2-4}$.

\section{Assistance cardiorespiratoire extracorporelle}

L'utilisation d'une approche veino-veineuse pour l'oxygénation extracorporelle par membrane (ECMO-VV) a été proposée comme option thérapeutique viable pour les patients atteints d'un SDRA grave. Cette approche était autrefois considérée comme un traitement de sauvetage pour le SDRA réfractaire, mais on dispose à présent de données suffisantes au sujet de son efficacité chez les patients atteints d'un SDRA grave dont l'état se détériore malgré l'optimisation des autres mesures thérapeutiques ${ }^{27-29}$. Chez ces patients, l'ECMO-VV peut servir de pont vers un rétablissement. Une canule fait passer le sang désoxygéné de la circulation systémique vers l'oxygénateur extracorporel à membrane qui l'enrichit en oxygène, en élimine le gaz carbonique sanguin et le retourne une fois oxygéné vers la circulation. Ce procédé extracorporel d'échanges gazeux permet de réduire la pression ventilatoire exercée sur le poumon malade, atténuant ainsi l'atteinte pulmonaire induite par le ventilateur dans les cas graves. Étant donné que l'ECMO est déjà un traitement valide, les médecins devraient sans tarder discuter de leurs cas potentiels avec les établissements de référence pour l'ECMO plutôt que d'y recourir en dernier ressort.

\section{Traitement pharmacologique}

Les corticostéroïdes ont été beaucoup étudiés pour le traitement pharmacologique du SDRA. En théorie, ils agissent en réduisant l'inflammation pulmonaire globale inhérente au SDRA et pourraient réduire le risque de mortalité dans les cas graves $^{24}$. Cela dit, leur utilisation chez les grands malades est également associée à d'importants effets indésirables, dont l'hypernatrémie, l'hyperglycémie et la faiblesse neuromusculaire. Cette dernière peut d'ailleurs avoir des effets dévastateurs chez les patients atteints d'un SDRA; les médecins doivent en tenir compte et soupeser ces risques potentiels.

Le traitement d'appoint par blocage neuromusculaire et sédation profonde peut aussi être envisagé chez les patients atteints d'un SDRA sous ventilation mécanique. Il est parfois difficile d'administrer des volumes courants faibles et réguliers chez un patient éveillé (souvent tachypnéique) qui respire spontanément, une situation appelée dyssynchronie patientventilateur ${ }^{32}$. C'est pourquoi la sédation profonde et le blocage neuromusculaire ont fait l'objet d'essais en association avec la ventilation mécanique pour le SDRA grave. Il est à noter que les résultats des études sur l'utilisation hâtive du blocage neuromusculaire chez les patients atteints de SDRA sont divergents $^{19,20}$. Cela dit, elle peut être envisagée pour optimiser l'oxygénation et la ventilation, même si elle n'est pas recommandée d'emblée chez tous les patients atteints d'un SDRA de modéré à grave ${ }^{21}$.

D'autres traitements pharmacologiques pour le SDRA ont aussi été mis à l'essai avec des résultats variables. Le maintien d'un équilibre liquidien conservateur allié à l'administration de diurétiques réduirait la durée de la ventilation mécanique et améliorerait la fonction pulmonaire chez les patients atteints d'un SDRA; il pourrait donc être bénéfique de généraliser la pratique $^{33}$. Par ailleurs, l'oxyde nitrique inhalé pourrait en théorie réduire la résistance vasculaire pulmonaire et la discordance ventilation-perfusion, même si les données randomisées ne révèlent aucun avantage sur le plan de la mortalité et suggéreraient même en fait une incidence négative ${ }^{25}$. Enfin, l'utilisation de la prostacycline en aérosol pour le SDRA a été étudiée ${ }^{26}$, mais il faudra approfondir la recherche sur ses effets avant d'en recommander l'utilisation systématique.

\section{Le SDRA associé à la COVID-19 est-il un syndrome distinct?}

La pandémie de COVID-19 a braqué les projecteurs sur le traitement du SDRA en 2020. Le SDRA secondaire à la COVID-19 grave a été (et est encore) fréquent; jusqu'à présent, on 
ignorait s'il s'agissait d'une entité distincte des autres formes de SDRA et s'il fallait appliquer des stratégies thérapeutiques différentes ${ }^{34}$. Après les premiers cas rapportés, on a réfléchi à d'autres façons d'intervenir en cas de SDRA associé à la COVID1935. Deux phénotypes distincts de SDRA ont été décrits chez les patients atteints de COVID-19 : le type $\mathrm{H}$, marqué par une élastance pulmonaire élevée, un rapport ventilation/perfusion élevé, un poids pulmonaire élevé et une forte recrutabilité alvéolaire (concordant avec le SDRA grave typique), et le type L, plus nouveau, marqué par des valeurs faibles des mêmes

Tableau 1 : Résumé des modalités de ventilation mécanique pour le syndrome de détresse respiratoire aiguë (SDRA) et recommandations des lignes directrices de pratique clinique de l'American Thoracic Society (ATS), de l'European Society of Intensive Care Medicine (ESICM), de la Society of Critical Care Medicine (SCCM), de la Société de réanimation de langue française (SRLF) et de l'Intensive Care Society (ICS)

\begin{tabular}{|c|c|c|c|c|c|}
\hline \multirow[b]{2}{*}{ Paramètre } & \multirow{2}{*}{$\begin{array}{l}\text { Gravité du } \\
\text { SDRA }\end{array}$} & \multirow[b]{2}{*}{ Raison d'être } & \multicolumn{3}{|c|}{ Force des recommandations } \\
\hline & & & ATS/ESICM/SCCM ${ }^{2}$ & SRLF $^{3}$ & $\mathrm{ICS}^{4}$ \\
\hline $\begin{array}{l}\text { Faibles volumes } \\
\text { courants ( } 4-8 \mathrm{~mL} / \mathrm{kg} \\
\text { de poids estimé) }\end{array}$ & Toutes & $\begin{array}{l}\text { La ventilation mécanique peut } \\
\text { potentialiser l'atteinte pulmonaire } \\
\text { aiguë, et la réduction des volumes } \\
\text { courants pourrait prévenir } \\
\text { l'atteinte induite par la ventilation }\end{array}$ & $\begin{array}{l}\text { Recommandation } \\
\text { forte pour } \\
\text { l'utilisation } \\
\text { systématique }\end{array}$ & $\begin{array}{l}\text { Accord fort pour } \\
\text { l'utilisation } \\
\text { systématique }\end{array}$ & $\begin{array}{l}\text { Recommandation } \\
\text { forte pour } \\
\text { l'utilisation } \\
\text { systématique }\end{array}$ \\
\hline $\begin{array}{l}\text { Pressions } \\
\text { respiratoires } \\
\text { moindres (pression } \\
\text { plateau }<30 \mathrm{~cm} \mathrm{H}_{2} \mathrm{O} \text { ) }\end{array}$ & Toutes & $\begin{array}{l}\text { Une pression plateau accrue peut } \\
\text { contribuer à l'atteinte pulmonaire } \\
\text { induite par la ventilation, même } \\
\text { en présence de volumes courants } \\
\text { appropriés }\end{array}$ & $\begin{array}{l}\text { Recommandation } \\
\text { forte pour } \\
\text { l'utilisation } \\
\text { systématique }\end{array}$ & $\begin{array}{l}\text { Accord fort pour } \\
\text { l'utilisation } \\
\text { systématique }\end{array}$ & $\begin{array}{l}\text { Recommandation } \\
\text { forte pour } \\
\text { l'utilisation } \\
\text { systématique }\end{array}$ \\
\hline $\begin{array}{l}\mathrm{PEP} \text { accrue et non } \\
\text { réduite }\end{array}$ & $\begin{array}{l}\text { Modéré/ } \\
\text { grave }\end{array}$ & $\begin{array}{l}\text { Une PEP accrue peut optimiser le } \\
\text { recrutement alvéolaire et réduire } \\
\text { le shunt intrapulmonaire, } \\
\text { atténuant ainsi l'atteinte }\end{array}$ & $\begin{array}{l}\text { Recommandation } \\
\text { conditionnelle pour } \\
\text { l'utilisation } \\
\text { systématique }\end{array}$ & $\begin{array}{l}\text { Accord fort pour } \\
\text { l'utilisation } \\
\text { systématique }\end{array}$ & $\begin{array}{l}\text { Recommandation } \\
\text { faible pour } \\
\text { l'utilisation } \\
\text { systématique }\end{array}$ \\
\hline
\end{tabular}

Décubitus ventral Grave Le décubitus ventral améliore le recrutement pulmonaire, principalement dans les régions dépendantes, ce qui accroît le volume expiratoire, améliore la concordance ventilation/perfusion et réduit l'atteinte pulmonaire induite par la ventilation

Ventilation oscillatoire à haute fréquence

Modéré/ grave

Méthode de ventilation qui fournit de très faibles volumes courants avec des pressions moyennes sur les voies respiratoires accrues, ce qui réduit le stress et la fatigue associés aux volumes courants

Manœuvres de

Toutes recrutement
Les manœuvres de recrutement (augmentation transitoire de la pression exercée sur les voies aériennes) peuvent réduire l'atélectasie et accroître le volume pulmonaire de fin d'expiration en ouvrant les alvéoles collabées

L'oxygénation extracorporelle et l'extraction du gaz carbonique peuvent remplacer la fonction pulmonaire dans les cas de SDRA, et permettre de régler le ventilateur aux valeurs minimales pour réduire l'incidence de l'atteinte pulmonaire induite par la ventilation
Accord fort pour

l'utilisation systématique (en forte pour

l'utilisation

systématique ( $>12 \mathrm{~h}$ par jour)

Recommandation forte - contre

l'utilisation systématique

Recommandation conditionnelle pour l'utilisation systématique

Absence de recommandation en raison des données insuffisantes au moment de rédiger la ligne directrice présence de $\mathrm{PaO}_{2} / \mathrm{FiO}_{2}$

$<150 \mathrm{~mm} \mathrm{Hg}$;

16 heures consécutives)

Accord fort - contre l'utilisation systématique

Accord fort - contre l'utilisation systématique

Accord fort - pour l'utilisation en cas de SDRA grave avec $\mathrm{PaO}_{2} / \mathrm{FiO}_{2}<80$ ou d'hypoxémie réfractaire
Recommandation forte - contre

l'utilisation systématique

Absence de recommandation en raison de données insuffisantes au moment de rédiger la ligne directrice

Recommandation faible chez certains patients 
variables ${ }^{36}$. Selon les experts, la plupart des patients atteints d'un SDRA associé à la COVID-19 présentent initialement des caractéristiques de type $L$, et quelques-uns seulement passent au type $\mathrm{H}$; par conséquent, les cliniciens devraient envisager une intubation précoce chez les patients présentant un SDRA de type L, car ces patients pourraient tolérer des volumes courants plus élevés sans risque d'atteinte pulmonaire induite par la ventilation ${ }^{35,36}$.

Cependant, un volume croissant de données probantes n'appuie pas cette caractérisation du SDRA associé à la COVID-1934. Premièrement, les patients non atteints de la COVID-19 qui répondent aux critères de la définition de Berlin du SDRA présentent déjà des degrés variables d'élastance pulmonaire et de recrutabilité ${ }^{5}$. De plus, l'idée de phénotypes distincts entre les patients atteints de SDRA a déjà été proposée et démontrée de manière plus rigoureuse $^{37}$. Pourtant, la mise en évidence de phénotypes ne s'est toujours pas traduite par des différences quant à la prise en charge médicale du SDRA ${ }^{34}$. Deuxièmement, d'autres évaluations de la mécanique respiratoire des patients atteints d'un SDRA associé à la COVID-19 montrent qu'elle est semblable à celle du SDRA classique ${ }^{38}$. En fait, les phénotypes proposés de SDRA associé à la COVID-19 correspondent probablement à l'évolution naturelle du SDRA. Par conséquent, les traitements existants fondés sur les données probantes qui misent sur la protection pulmonaire et la prévention des lésions iatrogènes représentent probablement la meilleure voie à suivre ${ }^{39}$. Même s'il est possible que de nouvelles données entraînent éventuellement une modification des approches thérapeutiques, aucune donnée probante convaincante n'indique pour l'instant que le SDRA associé à la COVID-19 est une entité distincte, ni qu'une stratégie thérapeutique différente s'impose, particulièrement en matière de ventilation. Les traitements d'usage courant utilisés pour le SDRA pourraient donc être efficaces pour le SDRA associé à la COVID-19.

Les patients atteints de la COVID-19 pourraient tirer des bienfaits d'une ventilation non effractive (par exemple par canule nasale à haut débit) et du décubitus ventral en état d'éveil, qui semblent tous les deux permettre d'améliorer l'hypoxémie et d'éviter l'intubation ${ }^{40,41}$ et qui font actuellement l'objet d'essais randomisés. Mentionnons particulièrement l'administration de corticostéroïdes (surtout la dexaméthasone), qui a permis de réduire les taux de mortalité chez les patients atteints de la COVID-19 sous ventilation mécanique $^{42,43}$. L'anticorps monoclonal tocilizumab pourrait être efficace pour réduire le recours à la ventilation mécanique et les décès chez les patients atteints de la COVID-19 hospitalisés ${ }^{44,45}$, mais l'anticoagulothérapie ne semble pas bénéfique chez les patients présentant une COVID-19 grave ${ }^{46}$. Enfin, de nombreux patients atteints d'un SDRA associé ou non à la COVID-19 pourraient nécessiter une ventilation mécanique de longue durée. Dans les cas où la trachéotomie est alors nécessaire; les cliniciens devraient suivre les recommandations concernant la sécurité des patients, les protocoles de trachéotomie et sa prise en charge ${ }^{47}$.

\section{Quelles incertitudes persistent au sujet de la prise en charge du SDRA?}

On résume à la figure 3 les principaux domaines d'étude d'intérêt en ce qui concerne le traitement du SDRA. Même si ce dernier repose principalement sur la ventilation associée à une approche de protection pulmonaire au moyen des réglages classiques de la pression ou du volume, de nouvelles stratégies ventilatoires pourraient aussi être efficaces. Tout d'abord, la ventilation avec relâchement de la pression des voies aériennes (APRV, pour airway pressure release ventilation) est un mode de ventilation impliquant un contrôle de la pression qui peut atténuer l'atteinte pulmonaire induite par le ventilateur. Cette approche permet de dégonfler périodiquement les poumons ("relâchement ») à partir d'un niveau plus élevé de pression positive continue, plutôt que d'essayer de gonfler les poumons à des volumes idéaux en contrant la piètre compliance par une augmentation de la pression. Théoriquement, en maintenant une pression continue à une intensité modérée, l'APRV pourrait réduire l'atteinte pulmonaire induite par le ventilateur. Cela dit, un récent essai randomisé a conclu que l'APRV n'avait aucune incidence sur les taux de mortalité par SDRA, même si elle a permis de réduire la durée de la ventilation mécanique et des séjours à l'USI comparativement à la ventilation avec volume contrôlé favorisant la protection pulmonaire ${ }^{48}$. D'autres essais cliniques sur l'efficacité de l'APRV seront nécessaires.

Le risque d'aggravation de l'atteinte pulmonaire par des lésions auto-infligées chez les patients est un autre domaine de recherche à approfondir. Même si la caractérisation de ce type d'atteinte s'appuie sur une solide explication physiologique, on dispose de très peu de données chez l'être humain pour bien le comprendre ${ }^{49}$. En théorie, on pourrait atténuer le risque d'atteinte pulmonaire auto-infligée en contrôlant le réflexe et les efforts respiratoires par un blocage neuromusculaire, la sédation ou l'assistance extracorporelle. À l'heure actuelle, on dispose toutefois de peu de données sur le lien entre le contrôle du réflexe et des efforts respiratoires et l'amélioration des issues pour les patients atteints d'un SDRA ${ }^{50}$.

Enfin, même si l'ECMO-VV est bénéfique pour les patients atteints d'un SDRA grave chez qui la prise en charge classique échoue, on dispose d'une autre forme nouvelle d'assistance extracorporelle qui pourrait être utile pour la prise en charge du SDRA modéré à grave : l'extraction de gaz carbonique au moyen d'une membrane de filtration extracorporelle ${ }^{51}$. Pour les patients sous ventilation mécanique qui ont de très faibles volumes courants, il y a un risque d'hypoventilation et d'hypercapnie ou d'acidose associées. L'extraction du gaz carbonique au moyen d'une membrane de filtration extracorporelle peut faciliter l'administration de très faibles volumes courants en offrant une méthode extracorporelle d'extraction du gaz carbonique. Comparativement à l'ECMO-VV, cette approche utilise de plus petits cathéters, même si les risques associés 
sont importants, surtout en raison des saignements ${ }^{52}$. L'extraction du gaz carbonique au moyen de la membrane de filtration extracorporelle peut être envisagée chez les patients qui ont un SDRA modéré et pourrait les empêcher de progresser vers le SDRA grave; des études sont en cours pour en analyser l'efficacité.

\section{Conclusion}

Le syndrome de détresse respiratoire aiguë provoque une insuffisance respiratoire qui découle le plus souvent d'une pneumonie, d'un sepsis, d'un trauma ou d'une aspiration. La présence d'une hypoxémie d'intensité croissante est

\section{Secteur}
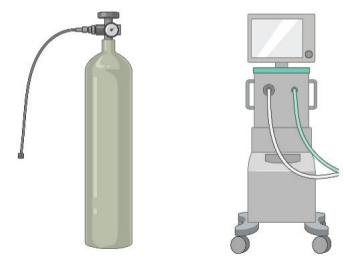

Stratégies de ventilation

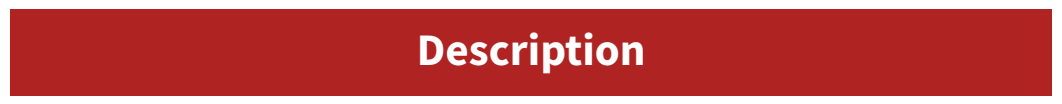

Stratégies d'oxygénation et de ventilation non effractives - L'oxygénothérapie par canule nasale à haut débit et la ventilation à pression positive non effractive par casque sont parfois utiles lors des premières phases du SDRA, car elles peuvent prévenir le recours à la ventilation mécanique effractive.

Stratégies de ventilation effractives - La ventilation avec relâchement de la pression des voies aériennes (APRV), le neuro-asservissement de la ventilation assistée (NAVA) ou la ventilation assistée proportionnelle (PAV) sont des modes de ventilation qui peuvent potentiellement atténuer le risque de dysfonction diaphragmatique associée au ventilateur et de dyssynchronie patient-ventilateur.

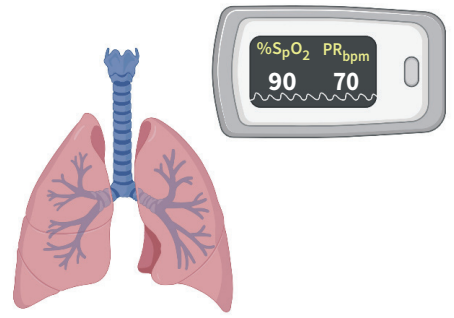

Cible de la ventilation mécanique - outils de surveillance
Respiration spontanée et effort inspiratoire - La surveillance et les interventions ciblant le réflexe $(\mathrm{P} 0.1)$ et les efforts $\left(\mathrm{P}_{\text {occ }}\right)$ respiratoires peuvent atténuer l'atteinte pulmonaire auto-infligée par le patient.

Recrutement - Des techniques comme la tomographie d'impédance électrique (TIE), le rapport recrutement/inflation (rapport R/I) et l'échographie pulmonaire peuvent aider à repérer les patients qui pourraient bénéficier du recrutement ou du décubitus ventral.

Intensité de la ventilation mécanique - Une pression et une puissance mécanique supérieures sont associées à un risque de mortalité plus élevé à n'importe quel moment de la ventilation mécanique jusqu'à 30 jours. II reste à déterminer si une intervention relative à ces variables se traduit par une amélioration de la survie.

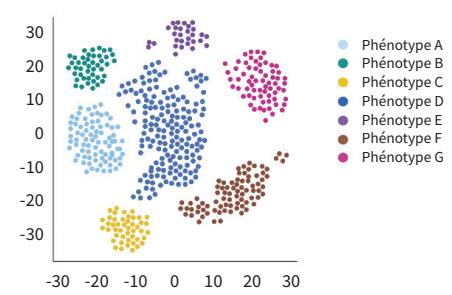

Phénotypes du SDRA
Hétérogénéité du SDRA - En tant que syndrome clinique, le SDRA inclut différentes entités ayant des pathophysiologies distinctes qui pourraient bénéficier de stratégies thérapeutiques adaptées.

Identification des phénotypes - L'étude des variables cliniques, de la mécanique respiratoire et des biomarqueurs pourrait mener à la définition de phénotypes secondaires présentant des caractéristiques biologiques communes, et ce qui pourrait permettre d'améliorer le pronostic et le traitement.

Analyse de l'air expiré - La mise en évidence de biomarqueurs dans l'air expiré pourrait être utile pour le diagnostic, le suivi de la trajectoire et le pronostic du SDRA chez les patients atteints.

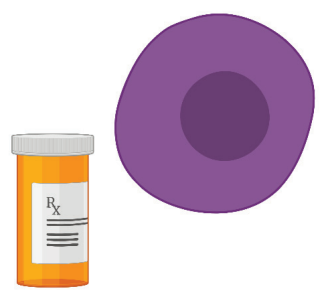

Traitements émergents
Immunomodulation - Des interventions pharmacologiques ciblant différentes voies de signalisation inflammatoire sont actuellement étudiées aux stades préclinique et clinique.

Cellules souches mésenchymateuses - Ces cellules médiatrices de la réparation pulmonaire par la libération de cytokines et de facteurs de croissance qui influent sur la réaction inflammatoire locale font l'objet d'études précliniques.

Extraction du $\mathrm{CO}_{2}$ extracorporelle - Un avantage clinique a été démontré chez les patients atteints d'un SDRA grave. La recherche se poursuit pour évaluer le rôle de l'extraction extracorporelle du $\mathrm{CO}_{2}\left(\mathrm{ECCO}_{2} \mathrm{R}\right)$ afin de faciliter la ventilation associée à une stratégie de protection pulmonaire.

Figure 3 : Orientations futures de la prise en charge du syndrome de détresse respiratoire aiguë (SDRA). 
associée à un risque élevé de mortalité. La prise en charge du SDRA passe principalement par le maintien des fonctions vitales, la ventilation avec stratégie de protection pulmonaire et la réduction des formes iatrogènes d'atteinte pulmonaire au moyen de modalités extracorporelles comme option pour les patients dont l'état continue de se détériorer malgré les mesures mises en place. Le syndrome de détresse respiratoire aiguë associé à la COVID-19 ne semble pas distinct du SDRA classique, et la prise en charge devrait se faire à l'aide des traitements existants.

\section{Références}

1. Fan E, Brodie D, Slutsky AS. Acute respiratory distress syndrome: advances in diagnosis and treatment. JAMA 2018;319:698-710.

2. Fan E, Del Sorbo L, Goligher EC, et al.; American Thoracic Society; European Society of Intensive Care Medicine; Society of Critical Care Medicine. An official American Thoracic Society/European Society of Intensive Care Medicine/Society of Critical Care Medicine clinical practice guideline: mechanical ventilation in adult patients with acute respiratory distress syndrome. Am J Respir Crit Care Med 2017;195:1253-63.

3. Papazian L, Aubron C, Brochard L, et al. Formal guidelines: management of acute respiratory distress syndrome. Ann Intensive Care 2019;9:69.

4. Griffiths MJD, McAuley DF, Perkins GD, et al. Guidelines on the management of acute respiratory distress syndrome. BMJ Open Respir Res 2019;6:e000420.

5. Bellani G, Laffey JG, Pham T, et al.; LUNG SAFE Investigators; ESICM Trials Group. Epidemiology, patterns of care, and mortality for patients with acute respiratory distress syndrome in intensive care units in 50 countries. JAMA 2016;315:788-800.

6. Laffey JG, Madotto F, Bellani G, et al.; LUNG SAFE Investigators; ESICM Trials Group. Geo-economic variations in epidemiology, patterns of care, and outcomes in patients with acute respiratory distress syndrome: insights from the LUNG SAFE prospective cohort study. Lancet Respir Med 2017;5:627-38.

7. Ashbaugh DG, Bigelow DB, Petty TL, et al. Acute respiratory distress in adults. Lancet 1967;2:319-23.

8. Bernard GR, Artigas A, Brigham KL, et al.; The American-European Consensus Conference on ARDS. Definitions, mechanisms, relevant outcomes, and clinical trial coordination. Am J Respir Crit Care Med 1994;149:818-24.

9. ARDS Definition Task Force; Ranieri VM, Rubenfeld GD, Thompson BT, et al. Acute respiratory distress syndrome: the Berlin Definition. JAMA 2012;307:2526-33.

10. Ferguson ND, Fan E, Camporota L, et al. The Berlin Definition of ARDS: an expanded rationale, justification, and supplementary material. Intensive Care Med 2012;38:1573-82.

11. Cochi SE, Kempker JA, Annangi S, et al. Mortality trends of acute respiratory distress syndrome in the United States from 1999 to 2013. Ann Am Thorac Soc 2016;13:1742-51.

12. Herridge MS, Cheung AM, Tansey CM, et al.; Canadian Critical Care Trials Group. One-year outcomes in survivors of the acute respiratory distress syndrome. N Engl J Med 2003;348:683-93.

13. Herridge MS, Tansey CM, Matté A, et al.; Canadian Critical Care Trials Group. Functional disability 5 years after acute respiratory distress syndrome. N Engl J Med 2011;364:1293-304.

14. Bellani G, Laffey JG, Pham T, et al.; LUNG SAFE Investigators; ESICM Trials Group. Noninvasive ventilation of patients with acute respiratory distress syndrome. Insights from the LUNG SAFE study. Am J Respir Crit Care Med 2017;195:67-77.

15. Amato MB, Barbas CS, Medeiros DM, et al. Effect of a protective-ventilation strategy on mortality in the acute respiratory distress syndrome. N Engl J Med 1998;338:347-54.

16. Acute Respiratory Distress Syndrome Network; Brower RG, Matthay MA, Morris $A$, et al. Ventilation with lower tidal volumes as compared with traditional tidal volumes for acute lung injury and the acute respiratory distress syndrome. N Engl J Med 2000;342:1301-8.

17. Briel M, Meade M, Mercat A, et al. Higher vs lower positive end-expiratory pressure in patients with acute lung injury and acute respiratory distress syndrome: systematic review and meta-analysis. JAMA 2010;303:865-73.
18. Guérin C, Reignier J, Richard J-C, et al.; PROSEVA Study Group. Prone positioning in severe acute respiratory distress syndrome. N Engl J Med 2013;368: 2159-68.

19. Papazian L, Forel JM, Gacouin A, et al.; ACURASYS Study Investigators. Neuromuscular blockers in early acute respiratory distress syndrome. $N$ Engl J Med 2010;363:1107-16.

20. National Heart, Lung, and Blood Institute PETAL Clinical Trials Network; Moss M, Huang DT, Brower RG, et al. Early neuromuscular blockade in the acute respiratory distress syndrome. N Engl J Med 2019;380:1997-2008.

21. Alhazzani W, Belley-Cote E, Møller MH, et al. Neuromuscular blockade in patients with ARDS: a rapid practice guideline. Intensive Care Med 2020;46:1977-86.

22. Young D, Lamb SE, Shah S, et al.; OSCAR Study Group. High-frequency oscillation for acute respiratory distress syndrome. N Engl J Med 2013;368:806-13.

23. Ferguson ND, Cook DJ, Guyatt GH, et al.; OSCILLATE Trial Investigators; Canadian Critical Care Trials Group. High-frequency oscillation in early acute respiratory distress syndrome. N Engl J Med 2013;368:795-805.

24. Ye Z, Wang Y, Colunga-Lozano LE, et al. Efficacy and safety of corticosteroids in COVID-19 based on evidence for COVID-19, other coronavirus infections, influenza, community-acquired pneumonia and acute respiratory distress syndrome: a systematic review and meta-analysis. CMAJ 2020;192:E756-67.

25. Adhikari NKJ, Burns KEA, Friedrich JO, et al. Effect of nitric oxide on oxygenation and mortality in acute lung injury: systematic review and meta-analysis. BMJ 2007;334:779

26. Fuller BM, Mohr NM, Skrupky L, et al. The use of inhaled prostaglandins in patients with ARDS: a systematic review and meta-analysis. Chest 2015;147:1510-22.

27. Combes A, Hajage D, Capellier G, et al.; EOLIA Trial Group; REVA; ECMONet. Extracorporeal membrane oxygenation for severe acute respiratory distress syndrome. N Engl J Med 2018;378:1965-75.

28. Goligher EC, Tomlinson G, Hajage D, et al. Extracorporeal membrane oxygenation for severe acute respiratory distress syndrome and posterior probability of mortality benefit in a post hoc Bayesian analysis of a randomized clinical trial [erratum publié dans JAMA 2019;321:2245]. JAMA 2018;320:2251-9.

29. Munshi L, Walkey A, Goligher E, et al. Venovenous extracorporeal membrane oxygenation for acute respiratory distress syndrome: a systematic review and meta-analysis. Lancet Respir Med 2019;7:163-72.

30. Slutsky AS, Ranieri VM. Ventilator-induced lung injury. N Engl J Med 2013;369: 2126-36.

31. Rochwerg B, Granton D, Wang DX, et al. High flow nasal cannula compared with conventional oxygen therapy for acute hypoxemic respiratory failure: a systematic review and meta-analysis. Intensive Care Med 2019;45:563-72.

32. Brochard L, Slutsky A, Pesenti A. Mechanical ventilation to minimize progression of lung injury in acute respiratory failure. Am J Respir Crit Care Med 2017;195:438-42.

33. National Heart, Lung, and Blood Institute Acute Respiratory Distress Syndrome (ARDS) Clinical Trials Network; Wiedemann HP, Wheeler AP, Bernard GR, et al. Comparison of two fluid-management strategies in acute lung injury. $N$ Engl J Med 2006;354:2564-75.

34. Fan E, Beitler JR, Brochard L, et al. COVID-19-associated acute respiratory distress syndrome: is a different approach to management warranted? Lancet Respir Med 2020;8:816-21.

35. Gattinoni L, Coppola S, Cressoni M, et al. COVID-19 does not lead to a "typical" acute respiratory distress syndrome. Am J Respir Crit Care Med 2020;201: 1299-300.

36. Gattinoni L, Chiumello D, Caironi P, et al. COVID-19 pneumonia: Different respiratory treatments for different phenotypes? Intensive Care Med 2020;46: 1099-102.

37. Calfee CS, Delucchi K, Parsons PE, et al.; NHLBI ARDS Network. Subphenotypes in acute respiratory distress syndrome: latent class analysis of data from two randomised controlled trials. Lancet Respir Med 2014;2:611-20.

38. Schenck EJ, Hoffman K, Goyal P, et al. Respiratory mechanics and gas exchange in COVID-19-associated respiratory failure. Ann Am Thorac Soc 2020;17:1158-61.

39. Alhazzani W, Evans L, Alshamsi F, et al. Surviving sepsis campaign guidelines on the management of adults with coronavirus disease 2019 (COVID-19) in the ICU: first update. Crit Care Med 2021;49:e219-34.

40. McEnery T, Gough C, Costello RW. COVID-19: respiratory support outside the intensive care unit. Lancet Respir Med 2020;8:538-9.

41. Thompson AE, Ranard BL, Wei Y, et al. Prone positioning in awake, nonintubated patients with COVID-19 hypoxemic respiratory failure. JAMA Intern Med 2020;180:1537-9. 
42. WHO Rapid Evidence Appraisal for COVID-19 Therapies (REACT) Working Group; Sterne JAC, Murthy S, Diaz JV, et al. Association between administration of systemic corticosteroids and mortality among critically ill patients with COVID-19: a meta-analysis. JAMA 2020;324:1330-41.

43. Siemieniuk RA, Bartoszko JJ, Ge L, et al. Drug treatments for COVID-19: living systematic review and network meta-analysis. BMJ 2020;370:m2980.

44. RECOVERY Collaborative Group; Horby PW, Pessoa-Amorim G, Peto L, et al. Tocilizumab in patients admitted to hospital with COVID-19 (RECOVERY): preliminary results of a randomised, controlled, open-label, platform trial. Lancet 2021;397:1637-45.

45. REMAP-CAP Investigators; Gordon AC, Mouncey PR, Al-Beidh F, et al. Interleukin-6 receptor anatagonists in critically ill patients with COVID-19. N Engl J Med 2021;384:1491-502.

46. Talasaz AH, Sadeghipour P, Kakavand H, et al. Antithrombotic therapy in COVID-19: systematic summary of ongoing or completed randomized trials. medRxiv 2021 Jan. 6. doi: 10.1101/2021.01.04.21249227.
47. McGrath BA, Brenner MJ, Warrillow SJ, et al. Tracheostomy in the COVID-19 era: global and multidisciplinary guidance. Lancet Respir Med 2020;8:717-25.

48. Zhou Y, Jin X, Lv Y, et al. Early application of airway pressure release ventilation may reduce the duration of mechanical ventilation in acute respiratory distress syndrome. Intensive Care Med 2017;43:1648-59.

49. Tobin MJ, Laghi F, Jubran A. P-SILI is not justification for intubation of COVID19 patients. Ann Intensive Care 2020;10:105

50. Spinelli E, Mauri T, Beitler JR, et al. Respiratory drive in the acute respiratory distress syndrome: pathophysiology, monitoring, and therapeutic interventions. Intensive Care Med 2020;46:606-18.

51. Del Sorbo L, Cypel M, Fan E. Extracorporeal life support for adults with severe acute respiratory failure. Lancet Respir Med 2014;2:154-64.

52. Boyle AJ, Sklar MC, MCNamee JJ, et al.; International ECMO Network; (ECMONet). Extracorporeal carbon dioxide removal for lowering the risk of mechanical ventilation: research questions and clinical potential for the future. Lancet Respir Med 2018;6:874-84.
Intérêts concurrents : Eddy Fan déclare avoir reçu des honoraires personnels d'ALung Technologies et de MC3 Cardiopulmonary, sans lien avec les travaux soumis. Aucun autre intérêt concurrent n'a été déclaré.

Cet article a été révisé par des pairs.

Affiliations : Division de médecine des soins intensifs (Fernando), Départements de médecine et de médecine d'urgence, Université d'Ottawa, Ottawa, Ont.; Division interdépartementale de médecine des soins intensifs (Ferreyro, Urner, Munshi, Fan), Université de Toronto; Institut pour les politiques, la gestion et l'évaluation de la santé (Ferreyro, Urner, Munshi, Fan), École Dalla Lana de santé publique, Université de Toronto; Faculté de médecine (Ferreyro, Urner, Munshi, Fan), Sinai Health System et Réseau universitaire de santé; Institut de recherche de l'Hôpital général de Toronto (Fan), Réseau universitaire de santé, Toronto, Ont.
Collaborateurs : Tous les auteurs ont contribué à l'élaboration et à la conception des travaux ainsi qu'à l'acquisition, à l'analyse et à l'interprétation des données. Tous les auteurs ont participé à la rédaction du manuscrit, en ont révisé de façon critique le contenu intellectuel important, ont donné leur approbation finale pour la version destinée à être publiée, et assument l'entière responsabilité de tous les aspects du travail.

Propriété intellectuelle du contenu : Il s'agit d'un article en libre accès distribué conformément aux modalités de la licence Creative Commons Attribution (CC BY-NC-ND 4.0), qui permet l'utilisation, la diffusion et la reproduction dans tout médium à la condition que la publication originale soit adéquatement citée, que l'utilisation se fasse à des fins non commerciales (c.-à-d., recherche ou éducation) et qu'aucune modification ni adaptation n'y soit apportée. Voir : https://creativecommons.org/ licenses/by-nc-nd/4.0/deed.fr.

Correspondance : Shannon Fernando, sfernando@qmed.ca 